\title{
Nature of Transport across Sheared Zonal Flows in Electrostatic Ion-Temperature-Gradient Gyrokinetic Plasma Turbulence
}

\author{
R. Sánchez, ${ }^{1, *}$ D. E. Newman, ${ }^{2}$ J.-N. Leboeuf, ${ }^{3}$ V. K. Decyk, ${ }^{4}$ and B. A. Carreras ${ }^{5}$ \\ ${ }^{1}$ Fusion Energy Division, Oak Ridge National Laboratory, Oak Ridge, Tennessee 37831-8071, USA \\ ${ }^{2}$ Department of Physics, University of Alaska, Fairbanks, Alaska 99775-5920, USA \\ ${ }^{3}$ JNL Scientific Inc., Casa Grande, Arizona 85294-9695, USA \\ ${ }^{4}$ UCLA, Los Angeles, California 90095-1547, USA \\ ${ }^{5}$ BACV Solutions Inc., Oak Ridge, Tennessee 37830-8222, USA
}

(Received 6 May 2008; published 12 November 2008)

\begin{abstract}
It is shown that the usual picture for the suppression of turbulent transport across a stable sheared flow based on a reduction of diffusive transport coefficients is, by itself, incomplete. By means of toroidal gyrokinetic simulations of electrostatic, collisionless ion-temperature-gradient turbulence, it is found that the nature of the transport is altered fundamentally, changing from diffusive to anticorrelated and subdiffusive. Additionally, whenever the flows are self-consistently driven by turbulence, the transport gains an additional non-Gaussian character. These results suggest that a description of transport across sheared flows using effective diffusivities is oversimplified.
\end{abstract}

DOI: 10.1103/PhysRevLett.101.205002

PACS numbers: 52.35.Ra, 05.40.Fb, 52.55.Fa, 52.65.Tt

It is widely accepted that the rate of the transport (of particles, energy, or any other quantity) carried out by turbulence can be significantly lowered by a perpendicular sheared flow [1]. Since these flows are intrinsically unstable against Kelvin-Helmholz instabilities, their sustainment requires additional stabilizing mechanisms, such as a magnetic field or rotation. These additional mechanisms also introduce waves, which can help drive the flows via the turbulent Reynolds stresses. For instance, drift waves may drive poloidal or toroidal (zonal) sheared flows in tokamak plasmas [2]. These zonal flows are central to the formation of the radial transport barriers characteristic of the enhanced regimes [3] in which the future International Thermonuclear Experimental Reactor tokamak will operate [4]. In atmospheric and oceanic flows, Rossby waves, driven by the change in rotation rate around the local vertical axis with latitude, play an analogous role [5]. The turbulent flux across the flow, $\tilde{\boldsymbol{\Gamma}}_{\perp}=\left\langle\tilde{s} \tilde{\mathbf{v}}_{\perp}\right\rangle$ (where $\tilde{s}$ is the advected quantity) can decrease due to a reduction in either the amplitude or the self-coherence of the turbulence [6], or to a shift in the phase between advected and advecting fields [7]. Importantly, the investigation (and modeling) of these various possibilities has traditionally been done by assuming from the start that some effective diffusivity characterizes transport in the absence of the flow, $D_{\perp} \sim$ $l_{\perp}^{2} / \tau$ ( $l_{\perp}$ and $\tau$ being typical transport scales), which is then reduced (via changes in $l_{\perp}$ and/or $\tau$ ) by the action of the sheared flow. Note, however, that the nature of transport across the flow must be and remain diffusive for this notion to be applicable. More precisely, this means that the dynamics must be and remain Gaussian and Markovian, so that a finite length $l_{\perp}$ and time $\tau$ exist. Otherwise, any description based on these ideas would provide an incomplete, even misleading, explanation. In this Letter we report on the first numerical evidence which suggests that the diffusive assumption is invalid in the presence of sheared zonal flows, driven either self-consistently by turbulence or externally, in magnetically confined toroidal plasmas. In particular, our simulations show that transport across them is subdiffusive for a large range of scales beyond the turbulent decorrelation time. Furthermore, the transport exhibits non-Gaussian features in the self-consistent case. Since transport suppression by stable sheared flows is a phenomenon of widespread occurrence in the earth and astrophysical sciences, the relevance of these results should transcend toroidal plasmas.

Our simulations have been carried out with the UCAN global gyrokinetic code [8]. UCAN evolves in time the nonMaxwellian part $(\delta f)$ of the ion distribution function of a collisionless deuterium plasma confined by a toroidal magnetic field with circular magnetic surfaces, coupled to the Poisson equation for the electrostatic potential $\phi$. Externally driven flows can be included by adding an additional external electric potential $\phi^{\text {ext }}(r)$. Since electrons are assumed to be adiabatic, UCAN is suitable to study the heat transported by ion-temperature-gradient (ITG) turbulence, but not to quantify particle transport. The geometry is a torus with major radius $R=1.7 \mathrm{~m}$ and minor radius $a=0.4 \mathrm{~m}$. Each location is labeled ( $r, \theta$, $\zeta$ ) being $r$ the radius normalized to $a$, and $\theta$ and $\zeta$ the poloidal and toroidal angles (see Fig. 1). The magnetic field has the safety factor profile shown in Fig. 1, and an axis value $B_{0}=1.87 \mathrm{~T}$. The fixed (in time) Maxwellian part of the ion distribution contains background density $\left(n_{0}\right)$ and ion temperature $\left(T_{0}^{i}\right)$ radial profiles such that $\eta_{i}(r) \equiv d\left(\ln T_{0}^{i}\right) / d\left(\ln n_{0}\right)$ has the profile shown in Fig. 1. At $r \simeq 0.4$, around which our studies will be carried out, the local values of some relevant parameters are $R / L_{T}=$ 5.25, $R / L_{n}=0.16, q=1.36$, and $\hat{s} \equiv(r / q)(d q / d r)=0.28$. Axis values for density and temperature $\left(T_{e} / T_{i}=1\right)$ are, 

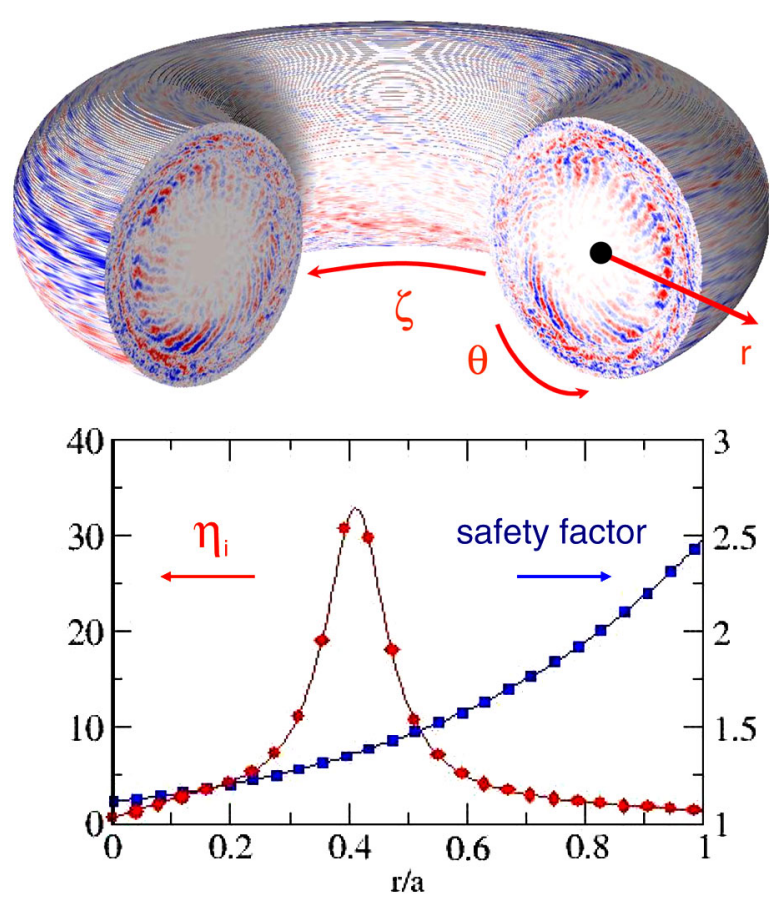

FIG. 1 (color online). Above: geometry of simulations discussed in text. Below: Safety factor [right, blue (or dark gray)] and $\eta_{i}$ [left, red (or gray)] profiles.

respectively, $3.1 \times 10^{19} \mathrm{~m}^{-3}$ and $0.7 \mathrm{keV}$ [similar values were used to compare with (the central $60 \%$ of) DIII-D tokamak discharges [9]]. Computations are done on a Cartesian grid of size $[X Y Z]=256 \times 256 \times 64$, periodic in $Z$ and including toroidal effects [8]. The radial size is $a / \rho_{i} \sim 200$. Time resolution is $d t=0.15 \mu \mathrm{s}$, much shorter than the turbulence decorrelation time $(\sim 10 \mu \mathrm{s})$.

UCAN is particularly useful for our purposes because it is a particle-in-cell code. It constructs $\delta f$ by pushing ions (in our runs: $\sim 3.4 \times 10^{7}$ or, equivalently, 8 particles-per-cell) carrying appropriate weights along the characteristic trajectories (in phase space) of the gyrokinetic equation [10]. The spatial part of these characteristics yields the gyroaveraged ion orbits. We use these orbits to compute the probability $P_{i}\left(r, t \mid r_{0}, t_{0}\right)$ of finding one ion at some radius $r$ and time $t$ if it was at another radius $r_{0}$ at time $t_{0} \leq t$, also referred to as the ion radial propagator. $P_{i}\left(r, t \mid r_{0}, t_{0}\right)$ can be used to probe the nature of radial transport (of real ions, not tracers) across sheared zonal flows, despite the fact that UCAN cannot quantify particle transport. To do this, we have run three simulations with the same parameters but using different zonal flow implementations. The first one is the usual one, with the ITG mode driving nonlinearly a poloidal and toroidal flow which acts back on the turbulence until both saturate [11]. In Fig. 2, the electrostatic energy for this case is shown in red (or gray). In the second simulation, the backreaction of the nonlinearly driven zonal flow is artificially suppressed by zeroing the surface average of the electrostatic potential (i.e., $\phi_{00}$ ) at every time step. Turbulence saturates at a higher energy level (in

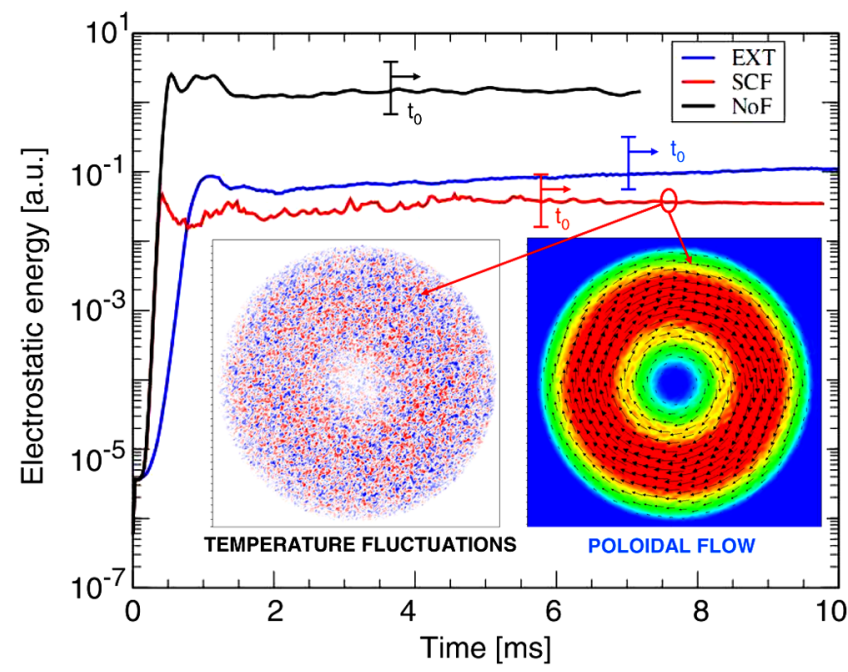

FIG. 2 (color online). Electrostatic energies for: no flow (black), self-consistent flow (red or gray), and externally driven flow [blue (or dark gray)]. $t_{0}$ (see text) marked with arrow. Inset: snapshot of toroidal cross sections of temperature fluctuations [positive $\rightarrow$ red (or gray), negative $\rightarrow$ blue (or dark gray)] and poloidal flow pattern [red (or gray) $\rightarrow$ strong, green (or light gray) $\rightarrow$ weak ].

black) since the zonal flow is absent, resulting in larger radial transport [12]. In the third run, $\phi_{00}$ is also zeroed out. But an external potential $\phi^{\mathrm{ext}}=\left\langle\phi_{00}\right\rangle_{\mathrm{nl}}$ is added. The brackets represent the time average over the saturated nonlinear phase of the first implementation. Since the externally driven flow has the same average strength and radial profile as the self-consistent one, the electrostatic energy [in blue (or dark gray)] saturates at a similar value.

We now discuss the ion radial propagators obtained in each of these cases. For the analysis to be meaningful, it is started at times well within the nonlinearly saturated phase. This is important as results from the nonstationary parts of the simulation may be misinterpreted as features characteristic of the transport dynamics. The starting times $\left(t_{0}\right)$ are marked with arrows in Fig. 2. We restrict the analysis to those ions present at $t_{0}$ within a narrow annulus (i.e., $r \in$ $[0.35-0.40])$, close to where the instability drive is maximum, and to elapsed times, $\Delta t \equiv t-t_{0}$, not too long but still larger than a meaningful number of eddy turnover times. In this way, two difficulties can be avoided. First, by avoiding the shortest times we avoid distortions of the propagator, for values of the radial displacement $(\Delta r \equiv$ $\left.r-r_{0}\right)$ up to the average banana width and elapsed times up to the mean inverse bouncing frequency $(\sim 0.2 \mathrm{~ms})$, caused by the fact that a fraction of the ions will follow trapped orbits [13]. Second, we avoid having to deal with the spatial inhomogeneities which would become apparent at long times if particles had time to move into regions in which the turbulence is very different. Typical propagators for the three cases are shown in Fig. 3 for $\Delta t$ values (shown using vertical lines in Fig. 4) well exceeding the trapped-ion distorted regime. The propagator variances 
$\left(\sigma^{2} \equiv\left\langle(\Delta r)^{2}-\langle\Delta r\rangle^{2}\right\rangle\right)$ are plotted vs $\Delta t$ in Fig. 4. The effect of trapped ions is apparent for the cases with zonal flows up to $\Delta t \sim 0.1-0.3 \mathrm{~ms}$. The distortion is, however, absent in the case with no zonal flow because the larger (unsuppressed) fluctuations kick ions out of their trapped orbits before they complete the banana.

To interpret these propagators, we first review some results from the theory of stochastic transport processes. It is well known that classical diffusion is the macroscopic consequence of microscopic Brownian motion. This statement can be proven, for instance, by constructing a random walk in which particles execute jumps of (typical) length $l$ after waiting at their locations for a (typical) time $\tau$ [The diffusivity is $D=l^{2} /(2 \tau)$.] Or, by formulating the Langevin equation for the position:

$$
x(t)=x_{0}+\int_{0}^{t} \xi_{2}\left(t^{\prime}\right) d t^{\prime} .
$$

$\xi_{2}(t)$ is Gaussian, uncorrelated noise [i.e., $\left\langle\xi_{2}(t) \xi_{2}\left(t^{\prime}\right)\right\rangle=$ $\left.2 D \delta\left(t-t^{\prime}\right)\right]$. Note that finite typical length and time scales are either explicitly (random walk) or implicitly (Langevin) assumed. The probability of finding one particle at $x$ at time $t$ if initially at $x_{0}$ can be found to be $(G[x]$ is a zero mean, unit variance Gaussian law):

$$
P_{\mathrm{Bm}}\left(x, t \mid x_{0}, 0\right)=G\left[\left(x-x_{0}\right) / \sqrt{D t}\right] .
$$

Thus, the propagator is a Gaussian centered at $x_{0}$ with variance growing as $t$. The connection with diffusion becomes clear when noting that Eq. (2) is also the solution of $\partial_{t} n=D \partial_{x}^{2} n$ that satisfies $n(x, 0)=\delta\left(x-x_{0}\right)$. Clearly, the UCAN results for the case with artificially suppressed zonal flow fit this description. The propagator (black, Fig. 3) is Gaussian and its variance (black, Fig. 4) scales as $\sigma^{2} \sim t$. Thus, the nature of turbulent radial transport is diffusive in the absence of the sheared zonal flow.

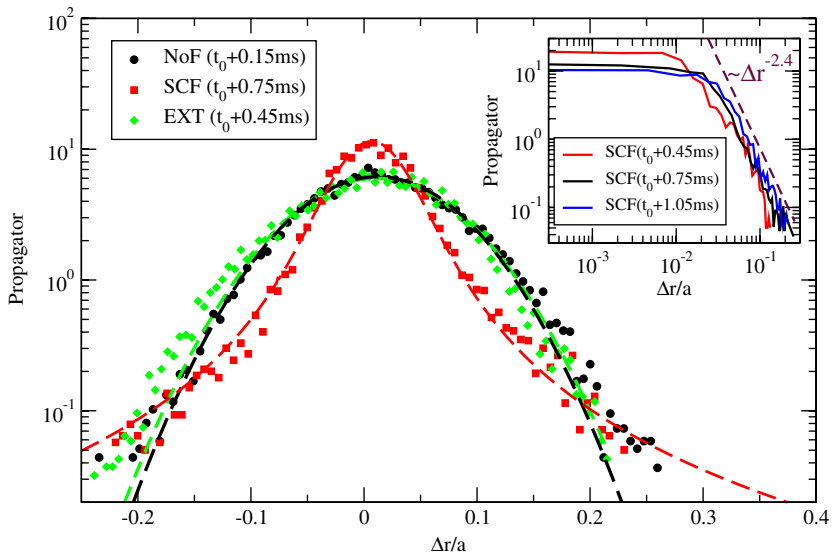

FIG. 3 (color online). Ion propagators for: no flow (black), self-consistent flow (red or gray) and externally driven flow [green (or light gray)]. Best fits shown in (same color or shading) dashed lines: Lévy fit in [red (or gray)] gives $\alpha=1.43$; other fits are Gaussian. Inset: ion propagator snapshots for self-consistent flow. Dashed line is not a fit.
This is not the case in the presence of the sheared zonal flow. If we examine first the externally driven zonal flow case, the propagator [green (or light gray), Fig. 3] is still Gaussian and the level of transport across the flow is reduced with respect to the case with suppressed zonal flow. But transport across the flow is no longer diffusive, since the variance (green or light gray, Fig. 4) grows as $\sim t^{0.5}$ for time scales exceeding both the turbulence decorrelation time and the ion bouncing time. This scaling implies subdiffusion, behavior typical of situations where finite transport scales are absent. The Langevin and random walk approaches must be generalized to deal with these cases, but their generalizations are no longer equivalent. The appropriate one here turns out to be the Langevin one:

$$
x(t)=x_{0}+\frac{1}{\Gamma\left(H-\frac{1}{\alpha}+1\right)} \int_{0}^{t} d t^{\prime}\left(t-t^{\prime}\right)^{H-1 / \alpha} \xi_{\alpha}\left(t^{\prime}\right),
$$

with $H \in(0,1]$ and $\alpha \in(0,2]$ and $\Gamma(x)$ the usual Euler's gamma function. For $\alpha=2$, this is the fractional Brownian motion ( $\mathrm{fBm}$ ) introduced by Mandelbrot [14], which reduces to the usual Brownian motion [(Eq. (1)] when $H=$ $1 / 2$. But in contrast to it, the successive increments $d x(t):=x(t+d t)-x(t)$ are correlated in time so that no finite typical time scale exists if $H \neq 1 / 2$. The fBm propagator can be found to be [15]:

$$
P_{\mathrm{fBm}}\left(x, t \mid x_{0}, 0\right)=G\left[(2 H)^{1 / 2} \Gamma\left(H+\frac{1}{2}\right) \frac{\left(x-x_{0}\right)}{D^{1 / 2} t^{H}}\right] .
$$

It is still a Gaussian, but $\sigma^{2} \sim t^{2 H}$. The motion is subdiffusive for $H<1 / 2$ and superdiffusive for $H>1 / 2$. Clearly, our externally driven flow results are consistent with $\mathrm{fBm}$ with $H=0.25 \pm 0.03$ within the intermediate time scales and up to the simulation end (strictly speaking, a diffusive scaling should be reached at long enough times [16], but these exceed the length of our simulations).

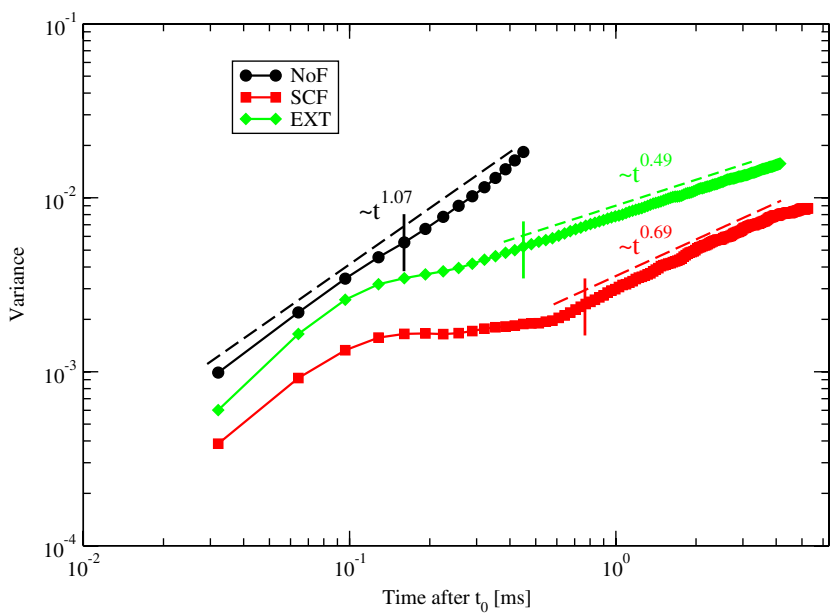

FIG. 4 (color online). Variance of ion propagators for: no flow (black), self-consistent flow [red (or gray)] and externally driven flow [green (or light gray)]. Vertical lines signal selected times for propagators in Fig. 3. 
Physically, it means that the ion radial velocities selfcorrelate over long times so that the probability of reversing their sign exceeds that of keeping it.

The case with the self-consistent zonal flow is different from the previous two. The propagator variance [red (or gray), Fig. 4] is subdiffusive (with $H \sim 0.34$ ) in the intermediate range, suggesting that a finite typical time scale is again absent. But it is not $\mathrm{fBm}$, since the propagator (red or gray, Fig. 3) is not Gaussian. Its convexity reverses so that it exhibits an algebraic tail that decays as $\sim(\Delta r)^{-2.4}$. Fat tails are characteristic of situations without a finite typical length scale $l$. They appear in the Langevin framework when considering $\alpha<2$ in Eq. (5), which means that the statistics of the uncorrelated noise $\xi_{\alpha}$ follow a symmetric Lévy form [17]. This choice, known as fractional Levy motion (fLm), is natural because Lévy laws also satisfy the central limit theorem which makes Gaussians preferential in nature. But they decay instead as power laws with exponent $1+\alpha, \alpha \in(0,2)$. Because of this, they have an infinite variance. In contrast, the finite variance of the Gaussian sets a finite typical length scale $l^{2} \propto \sigma^{2}$. The fLm propagator is [15] $\left(L_{\alpha}[x]\right.$ is a symmetric Lévy law of index $\alpha$ and scale factor unity):

$$
P_{\mathrm{fLm}}\left(x, t \mid x_{0}, 0\right)=L_{\alpha}\left[(\alpha H)^{1 / \alpha} \Gamma\left(H-\frac{1}{\alpha}+1\right) \frac{\left(x-x_{0}\right)}{D^{1 / \alpha} t^{H}}\right] .
$$

It reduces to $\mathrm{fBm}$ if $\alpha=2$, and to Brownian motion if in addition $H=\frac{1}{2}$. The motion is uncorrelated only when $H=1 / \alpha$. Its finite moments have order $s<\alpha$ and grow as $t^{s H}$. But the variance can still be computed in a finite system. It scales as in the Gaussian case, $\sigma^{2} \sim C t^{2 H}$, but $C$ diverges now with system size and/or life span. Most interestingly, the non-Gaussian ion propagator of our self-consistent flow case is fitted extremely well by a symmetric Lévy law with $\alpha=1.43$ [red (or gray), Fig. 3]. In fact, by carrying out this fit for a sequence of successive $\Delta t$ 's one concludes that the results are consistent with fLm with $\alpha=1.42 \pm 0.04$ and $H=0.34 \pm 0.03$.

In summary, we have shown that the nature of turbulent transport across stable sheared zonal flows is not diffusive but exhibits instead subdiffusive features within a range of scales which extends well beyond local turbulent time scales. In the case of self-consistent zonal flows, it appears to become non-Gaussian as well. Although this phenomenology is robust, the actual value of the exponents $\alpha$ and $H$ depends on the strength and length scale of the shear of the flow. The weaker the shear, the more diffusivelike transport will behave, which might be consistent with previous results reported by Lin and Hahm [18], although a more advanced analysis than theirs is needed to characterize the nature of transport. Our findings should have an important practical impact in quantifying transport across sheared flows for time scales over which subdiffusion dominates. They also point to the need for revisiting past fluid simu- lations [19] in which, in contrast to the supercritical ones examined here, the plasma profiles wandered around marginality for much longer times than what gyrokinetic codes can currently do. These simulations provided a view of the long-term transport dynamics dominated by superdiffusive transport, but included no zonal flows. Although it is probable that the long-term dynamics will remain qualitatively unchanged, our results show that a proper zonal flow evolution is essential to capture the dynamics throughout all scales. Because of the generality of the suppression mechanism via sheared flows, our conclusions should be applicable beyond the context discussed here, including astrophysical, atmospheric, and oceanic stable sheared flows. Finally, it is worth noting that the fact that $\mathrm{fBm}$ and fLm fit so well the radial propagators from our simulations elegantly connects with recent theoretical work showing that, under similar assumptions for a prescribed turbulent flow, the transport of any passive quantity becomes nondiffusive and can be analytically obtained in terms of fractional differential equations [16].

Research carried out at ORNL, managed by UT-Battelle LLC, for US DOE under Contract No. DE-AC0500OR22725. Research funded by DOE Office of Science Grants No. DE-FG02-04ER54741 at University of Alaska and No. DE-FG02-04ER54740 at UCLA. Simulations run at DOE's NERSC and University of Alaska's Arctic Region Supercomputing Center.

*Corresponding author. sanchezferlr@ornl.gov

[1] P. W. Terry, Rev. Mod. Phys. 72, 109 (2000).

[2] P. H. Diamond, S.-I. Itoh, K. Itoh, and T. S. Hahm, Plasma Phys. Controlled Fusion 47, R35 (2005).

[3] F. Wagner et al., Phys. Rev. Lett. 49, 1408 (1982).

[4] E. J. Doyle et al., Nucl. Fusion 47, S18 (2007).

[5] A. E. Gill, Geophys. Astrophys. Fluid Dyn. 6, 29 (1974).

[6] H. Biglari and P. H. Diamond, Phys. Fluids B 2, 1 (1990).

[7] P. W. Terry et al., Phys. Rev. Lett. 87, 185001 (2001).

[8] R. D. Sydora, V.K. Decyk, and J. M. Dawson, Plasma Phys. Controlled Fusion 38, A281 (1996).

[9] C. L. Rettig et al., Phys. Plasmas 8, 2232 (2001).

[10] T. S. Hahm, Phys. Fluids 31, 2670 (1988).

[11] Z. Lin et al., Science 281, 1835 (1998).

[12] J. N. Leboeuf et al., Phys. Plasmas 7, 1795 (2000).

[13] F. Hinton et al., Rev. Mod. Phys. 48, 239 (1976).

[14] B. Mandelbrot and J. van Ness, SIAM Rev. 10, 422 (1968).

[15] N. Laskin et al., Comput. Netw. 40, 363 (2002); I. Calvo et al., J. Phys. A 41, 282002 (2008).

[16] R. Sánchez et al., Phys. Rev. E 74, 016305 (2006).

[17] G. Samorodnitsky and M. S. Taqqu, Stable Non-Gaussian Processes (Chapman \& Hall, New York, 1994).

[18] Z. Lin and T. S. Hahm, Phys. Plasmas 11, 1099 (2004).

[19] B. A. Carreras et al., Phys. Plasmas 8, 5096 (2001); D. delCastillo-Negrete et al., Phys. Plasmas 11, 3854 (2004). 